\title{
Harmonicity of Sound Alters Roughness Perception
}

\author{
Didem Katircilar ${ }^{1,2}$ and Funda Yildirim ${ }^{1,2}$ \\ ${ }^{1}$ Cognitive Science Program, Yeditepe University, Istanbul, Turkey \\ ${ }^{2}$ Computational Neuroscience and Visual Perception Laboratory, Department of Computer Engineering, \\ Yeditepe University, Istanbul, Turkey
}

Multisensory integration refers to the integration of multiple senses by the nervous system. Auditory and tactile features are closely related senses as can be understood from the fact that adjectives such as soft, rough, and warm are used commonly for auditory and tactile features. Previous studies show different characteristics of auditory cues may cause perceiving surface rougher or smoother. In this study, we investigate the effects of harmonic and inharmonic sounds on roughness perception to examine whether auditory roughness will affect the tactile roughness perception while they are presented simultaneously. We expected the participants to perceive surfaces rougher while they listen to inharmonic sounds due to auditory roughness. We presented simultaneous and sequential harmonic and inharmonic sounds with three sandpapers with different roughness levels (P100, P120, P150 grit numbers) to the participants. We found that participants perceive sandpaper with the P120 grit number rougher while they listen to simultaneous inharmonic sounds than simultaneous harmonic sounds. However, any effect of harmonicity on the sandpapers with P100 and P150 grit numbers was not observed. We suggest that auditory roughness may enhance tactile roughness perception for surfaces with particular roughness levels, possibly when there is an ambiguity in roughness estimation.

Keywords: Roughness perception, audio-tactile perception, multisensory integration, tactile roughness, auditory roughness, harmonicity

\section{Introduction}

Our cognitive capacity allows us to process more than one kind of sensory information while perceiving external events (Stevenson et al., 2014). This information from different sensory modalities may be combined in our brain to create a strong perception (Ernst \& Di Luca, 2011). This process is called 'multisensory integration,'. In some cases, the information from one sensory modality may also affect the salience of stimulus from the other sensory modality which corresponds to 'cross-modal interaction' (Spence, 2011). Combined sensory inputs may create a 'unitary perception' in multisensory integration whereas altering the salience of sensory stimuli may improve or impair the perception in cross-modal interaction (Stein \& Stanford, 2008). Texture perception is a cross-modal experience with extensive practical applications, which range from the trivial, like evaluating clothes, to the serious, like diagnosing abnormal tissues. Most studies that investigate texture perception focus on roughness perception since it is a crucial factor to determine the physical or perceptual features of a surface (Altinsoy, 2008). People can assess the roughness of a surface by rubbing their finger on it (Altinsoy, 2008) since one can intuitively feel the texture properties of an object touching it (Suzuishi et al., 2020).

Although surface roughness perception is generally in the scope of tactile modality, the visual and auditory exposure from the surfaces during roughness estimation experience makes it a multimodal process (Altinsoy, 2008; Klatzky \& Lederman, 2010). Some studies have investigated how touch-produced sounds affect our 
tactile roughness perception (Guest et al., 2002; Lederman \& Klatzky, 1999). Lederman (1979) claimed that people are capable of distinguishing the sounds which are generated by touching different surfaces when they were given to the participants in isolation. However, further experiments showed that when both auditory and tactile cues are available, participants rely on tactile cues to determine surface roughness. Roughness perception is almost completely dominated by tactile cues because touching rarely produces significant enough sounds in our daily lives (Altinsoy, 2008). The effects of auditory stimuli were also tested for in visual and tactile roughness discrimination studies and these studies similarly showed that auditory stimuli did not improve the roughness perception (Heller, 1982). However, when participants use rigid probes, which create louder sounds than fingertips while exploring surfaces, they use both auditory and tactile texture cues to determine surface roughness. To investigate the effect of loudness of the auditory stimuli on roughness perception, Altinsoy (2008) presented the touch produced sound $10 \mathrm{~dB}$ higher than their actual value, and as in Lederman and Klatzky's (1999) research; participants consider auditory cues besides tactile stimuli for roughness perception. These studies showed that a sound's different characteristics may play a crucial role in cross-modal interaction. For example, Jousmaki and Hari (1998) changed the frequencies and amplitudes of the sounds of rubbing palms together. This study showed that people are likely to report higher smoothness and dryness when they are subjected to sounds with higher frequencies and amplitudes. Similarly, Guest et al. (2002) indicated that people judge abrasive surfaces to be rougher when they are subjected to sounds with higher frequencies.

Besides studies that consider how touch-produced sounds affect tactile texture and roughness perception, there are other studies that investigate how different sound characteristics of non-touch-produced sounds affect tactile perception. Suzuki et al.'s study (2008) showed that synchronizing finger movements with sound intensity causes impairment in roughness estimation whereas it had no effect on length estimation. These results show that auditory and tactile roughness processing may be based on common mechanisms (Suzuki et al., 2008). Further research also indicated that white noise and pure tones had an impact on perception of coarse surfaces but not on the perception of fine surfaces. White noise caused perceiving coarse surface pairs rougher whereas pure tones caused perceiving them smoother (Suzuki et al., 2008).

Furthermore, there are low-level psychoacoustic features such as auditory roughness that may have an impact on cross-modal processing of roughness perception (Liew et al., 2017). It is hypothesized that low-level characteristics of music can be related to features of other sensory systems. There are some examples of this interaction between senses. For instance, a higher pitch is matched with sweetness and sourness whereas a lower pitch is matched with bitterness in taste perception (Crisinel \& Spence, 2010). Major and minor keys have an impact on the perception of colors such as yellow and blue (Palmer et al., 2013; Lindborg \& Friberg, 2015). Ramachandran and Hubbard (2001) refer to a perceptual phenomenon which is called synaesthesia to explain the interaction of senses. On the other hand, Deroy et al. (2013) explain these kinds of associations in the brain by organization of the perceptual system. They claimed that innate neural connections or common coding between different sensory mechanisms may result in cross-modal pairings. For instance, size may be processed in the same way as loudness in our brain since both of them are related to magnitude (Deroy et al., 2013). In Liew et al.'s study (2017), participants tended to perceive dissonant sounds as spiky and rough and matched these sounds with rougher objects whereas less dissonant sounds were matched with the smoother objects (Liew et al., 2017). They argue that dissonance perception and audio-visual cross-modal interactions take place preconsciously and they are both low-level processing. 
Besides psychophysical studies, there are neuroimaging and electrophysiological studies investigating whether cross-modal processing takes place in low-level or high-level brain areas (Xu et al., 2020). Although previous research had indicated that high-level brain areas such as the superior temporal sulcus or posterior parietal cortex (PPC) are responsible for sensory interactions (Yau et al., 2015), later research on low-level brain areas such as primary sensory cortices (Ghazanfar et al., 2005; Lakatos et al., 2007), brainstem (Koehler et al., 2011) or thalamus (Komura et al., 2005; Bieler et al., 2018) showed that cross modal processing already occurs at early stages. As far as audio-tactile interaction is concerned, caudomedial auditory association cortex is involved in both somatosensory and auditory processing. Together, these results show that multisensory integration may take place at the early level of auditory cortical processing for both auditory and somatosensory sensory inputs (Schroeder et al., 2001). Tactile, somatosensory and auditory systems are also considered as associated systems due to the frequency-based neural codes of these two modalities (Ro et al., 2013, Butler's et al., 2012). Superior temporal gyrus as well as parietal and temporal regions in general are parts of the brain where auditory and somatosensory inputs are combined (Foxe et al., 2002, Pérez-Bellido et al., 2018). However, whether audio-tactile interaction requires low-level or high-level cognitive processing with respect to roughness perception is still an open question.

In the light of previous findings, we investigated how people obtain auditory information from harmonic and inharmonic sounds produced simultaneously or sequentially (similar to chord and arpeggio) and how these affect tactile roughness perception. Research shows that inharmonic tones are perceived to be rough (Carroll, 1970). Therefore, we hypothesized that participants would perceive surfaces to be rougher while they are listening to inharmonic sounds compared to harmonic sounds since auditory roughness is expected to enhance tactile roughness perception due to cross-modal audio-tactile interaction. Moreover, we expected to observe a stronger effect of audio-tactile interaction in simultaneously presented harmonic and inharmonic sounds than sequentially presented sounds. This is because sound waves, including simple ratio frequencies, are resolved better in the subcortical auditory system when they are presented simultaneously (Tramo et al., 2001, Bidelman and Heinz, 2011) whereas sequentially presented sounds are stored and maintained in working memory and require higher-level categorization (Tezcan-Semerci, 2020).

\section{Method}

\section{Participants}

There were 40 healthy participants with an age range of 18-34 (39 right-handed, 19 female, mean age: $24.375, \mathrm{SD}=4.505$ ) in this study. They did not report any loss of sense for their tactile and auditory senses and also did not have professional music history. This experiment was approved by Yeditepe University Ethical Committee for Social Sciences and was in compliance with the WMA Declaration of Helsinki.

\section{Stimuli}

Auditory Stimuli: Eight different sound stimuli were used in this experiment. Four of these stimuli were harmonic sounds and the other four were inharmonic sounds. Every sound consisted of three frequencies. Two harmonic sounds were generated by using A3 and A4 notes as fundamental frequencies and their first and second harmonics respectively. The inharmonic version of harmonic sounds was generated by adding a 
constant amount ( $30 \mathrm{hZ}$ ) to all frequencies (Schneider \& Frieler, 2008). Harmonic and inharmonic sounds were separated into two groups as sequential and simultaneous harmonic and inharmonic sounds.

Simultaneous and sequential harmonic and inharmonic sounds were generated by using the same frequencies (see Table 1). However, simultaneous sounds were created by overlapping all the frequencies while sequential sounds were generated by adding all the frequencies one after the other. VoceVista Pro was used to create sounds with specific frequencies and the piano effect was used. Sequential and simultaneous sounds were arranged by using Audacity.

Table 1

Sound Frequencies (hZ)

\begin{tabular}{ccc}
\hline & Harmonic & Inharmonic \\
\hline Sequential & $130.8-261.6-392.4$ & $160.8-291.6-422.4$ \\
Sequential & $220-440-660$ & $250-470-690$ \\
Simultaneous & $130.8-261.6-392.4$ & $160.8-291.6-422.4$ \\
Simultaneous & $220-440-660$ & $250-470-690$ \\
\hline
\end{tabular}

Tactile Stimuli: Sandpapers with three different grit numbers (P100, P120,P 150) and a surface made of PVC (polyvnyl chloride) were used as tactile stimuli. All surfaces were 8 × $8 \mathrm{~cm}$. Sandpapers and a PVC surface were placed on a board (See Figure 1).

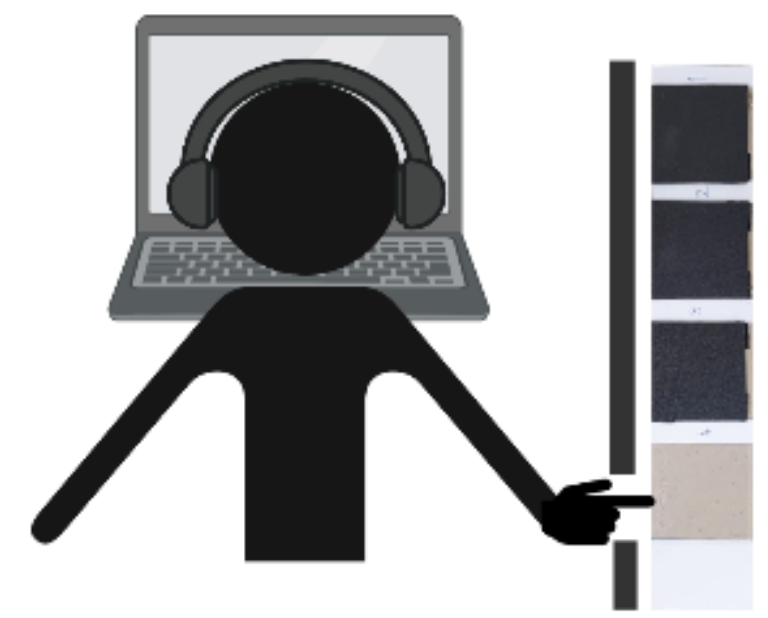

Figure 1: In the experimental setup participants touch different surfaces behind the standing screen with their index finger while listening to either harmonic or inharmonic sounds or hearing no sound at all. Tactile stimuli are sandpapers with P100, P120, P150 grit numbers and PVC surface (created with BioRender.com).

\section{Procedure}


All participants sat on a chair and stretched their arms through a hole on the standing screen to touch surfaces. They received all their instructions from the screen. Auditory stimuli were presented by headphones (Sony ZX110NC) with $50 \mathrm{~dB}$ intensity. Standing screen was used to prevent visual cues. Participants were asked to touch the surface when the auditory stimuli began and stop touching the surface when the auditory stimuli ended. All participants touched the surfaces by moving the fingertip of their dominant hand from right to left. In addition to this, there was a control condition in which three sandpapers were presented without any auditory stimuli. In this condition, participants started to touch when they heard a beep sound and they continued their exploration until they heard a second beep. They kept their headphones on to block sounds that are generated by touching. In both experimental and control conditions, they touched the surfaces for 6 seconds. Participants rated the surface roughness on a scale between 1 (from smooth) 9 (to rough). In addition to that, after participants touched sandpapers three times, they were subsequently asked to touch a PVC surface for 5 seconds to prevent habituation to sandpaper.

Participants were asked to stabilize their speed and pressure for each trial while they were moving their fingertip on the surface to make sure that they explored each surface in the same way. After every two participants, surface materials were replaced to make sure that they do not get flattened due to repeated touching.

Every harmonic and inharmonic sound was matched with a particular type of surface exactly once in the experiment. A surface was presented 8 times in total. The presentation of the surfaces was pseudorandomized.

\section{Statistical Analysis}

We computed the mean roughness score i.e. roughness rating for each type of stimuli in the experiment. Repeated-Measures ANOVA analyses were performed to investigate whether harmonic and inharmonic sounds affect perceived roughness and whether sequential and simultaneous sounds show a significant difference for tactile roughness perception. We also performed the simple main effect analyses for every surface separately to examine the effects of harmonic and inharmonic sounds on tactile roughness perception.

We also performed one sample t-tests for the roughness scores of the three surface types in sound and nosound conditions in order to test whether the presence sound — regardless of characteristics - alters roughness perception.

We conducted all statistical analyses with open-source JASP software (Love et al., 2019).

\section{Results}

Repeated Measure ANOVA indicated that harmonicity had a main effect on tactile roughness perception $(\mathrm{p}=0.047, \eta 2 \mathrm{p}=0.049)$. Moreover, simultaneous and sequential sounds showed a significant difference in tactile roughness perception when considering all paper types $(\mathrm{p}=0.047, \eta 2 \mathrm{p}=0.049)$ (see Figure 2$)$. There was also an interaction between paper type and harmonicity $(\mathrm{p}=0.033, \eta 2 \mathrm{p}=0.042)$. Simple main effect analysis indicated that for simultaneous sounds, inharmonic sounds $(\mathrm{M}=6.162, \mathrm{SD}=1.892)$ led to perceive sandpaper with $\mathrm{P} 120$ grit number rougher than harmonic sounds $(\mathrm{M}=6.787, \mathrm{SD}=1.927)(\mathrm{p}=0.002)$. However, for sandpapers with P100 and P150 grit numbers, harmonicity had no effect. 
In addition, participants successfully discriminated the roughness of each sandpaper ( $<<0.001$, see Figure 3 ).

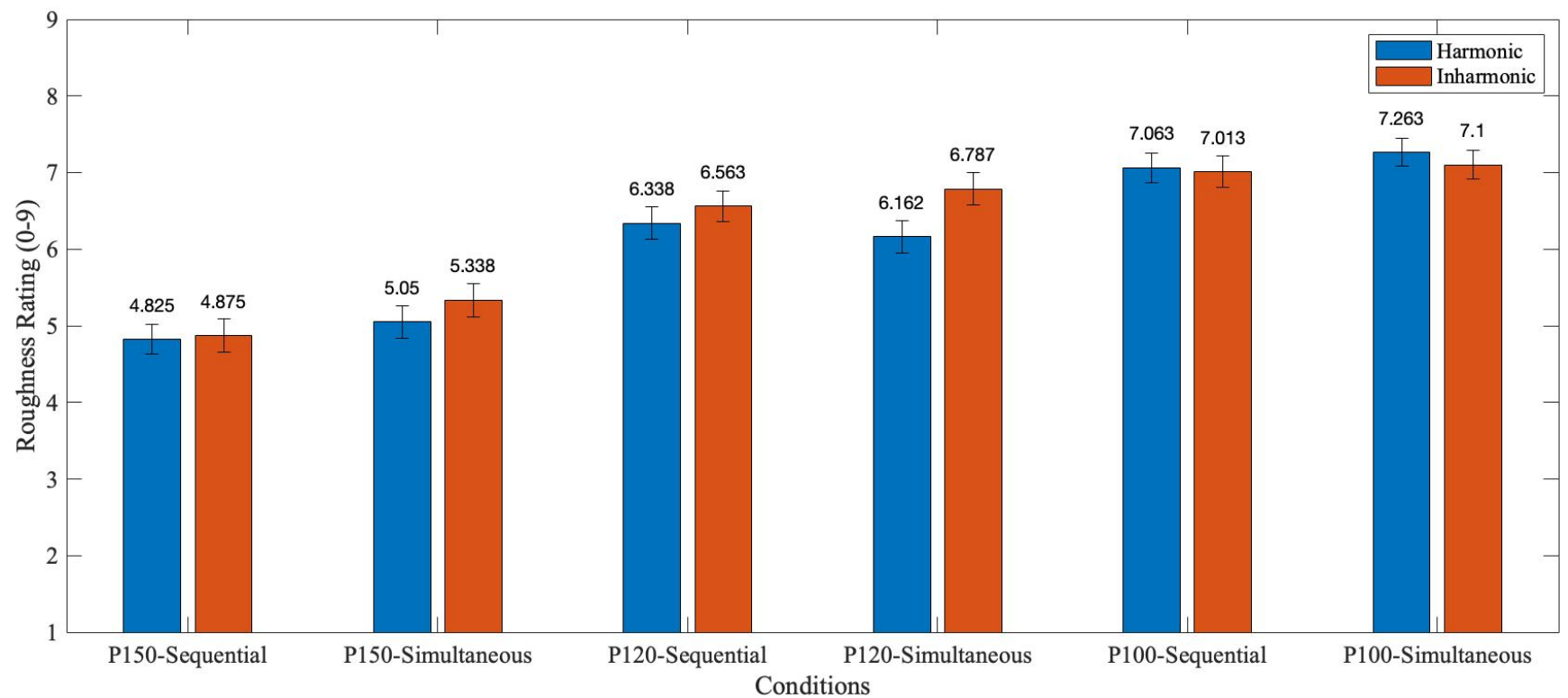

Figure 2: Participant ratings for harmonic and inharmonic sound conditions for all sandpaper types (bars indicate standard errors). 


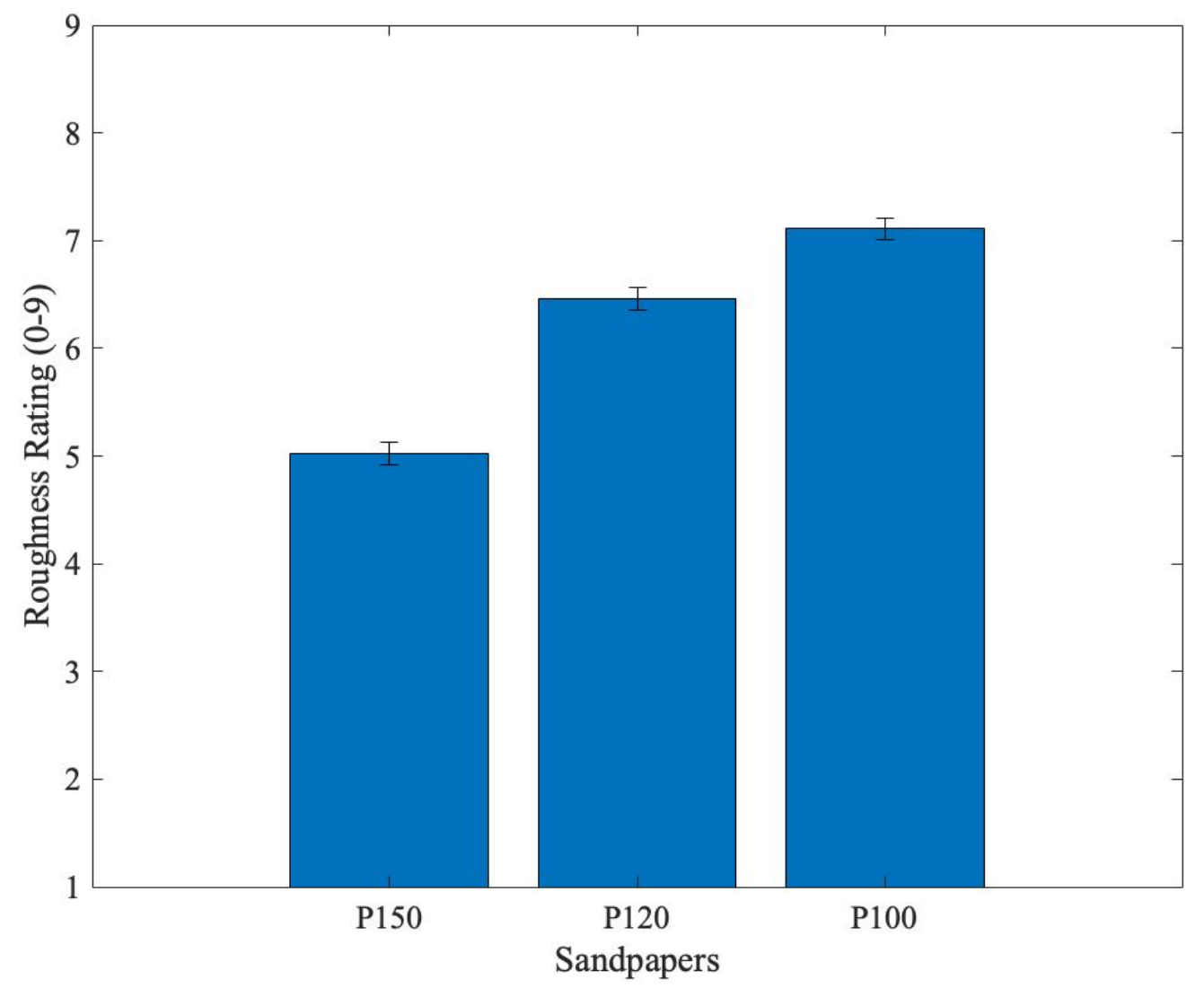

Figure 3: Participant ratings given to the sandpapers with P150, P120 and P100 grit numbers for roughness with standard error bars.

One sample t-tests results showed that participants' perceived roughness is higher in no sound condition than sound condition for sandpaper with P150 grit numbers. For sandpaper with P120 grit number, participants' perceived roughness is higher in sound condition than no sound condition. There is no statistically significant difference for sandpaper with P100 grit number.

\section{Discussion}

Besides touch-generated sounds, sounds and surfaces that have corresponding properties such as roughness may affect our tactile perception. This study demonstrates that low-level sound characteristics of the auditory stimuli such as harmonicity can affect tactile roughness perception. We expected inharmonic sounds - which are described to have auditory roughness - to make surfaces appear rougher compared to harmonic sounds. As expected, paper type, harmonicity and presentation of sounds as sequential and simultaneous have a main 
effect on participants' perceived roughness. Participants could discriminate between smooth, medium and rough surfaces. Moreover, they perceived the surface with P120 grits which has medium level roughness among our sandpapers significantly rougher, when they listened to simultaneous inharmonic sounds rather than simultaneous harmonic sounds. However, there is no statistically significant difference in roughness perception for sandpapers with P100 and P150 grit numbers. Although further research is required to explain why the auditory roughness affected the perceived surface roughness for only the sandpaper with P120 grit number; some of the participants gave feedback about the roughness discrimination of the surfaces after the experiment. The participants thought that there were three different surfaces. One surface was the roughest, the other the smoothest, and then there was the PVC surface. It is reasonable to expect for participants to have a hard time discriminating between the sandpaper with P120 grit number from either the roughest sandpaper (P100 grit number) or the smoothest sandpaper (P150 grit number). These results may support the argument that both auditory and tactile modalities are used together in this challenging roughness discrimination task. In Chimata and Schwartz (2018), when three sandpapers of different grit numbers (P800, P1200, P1500) were presented to participants as pairs, the participants could discriminate the difference between sandpapers with the highest and lowest grit numbers (P800-P1500) better compared to sandpaper pairs such as P800-P1200 and P1200-P1500. Based on the participants' feedback after our experiment and previous research such as Chimata and Schwartz (2018), it is likely that participants relied on tactile input while deciding the roughness of the surface with P150 and P100 grit numbers. However, people may tend to integrate available sensory stimuli when faced with ambiguous tactile stimuli in order to create a robust perception (Ernst \& Bülthoff, 2004; Murai \& Yotsumoto, 2018). Since the roughest and the smoothest tactile stimuli were different enough, participants did not need to consider auditory cues to discriminate roughness.

Moreover, Parncutt and Hair (2011) claimed that frequencies that can not be separated easily are related to auditory roughness which could be exemplified as rubbing your fingertip on a sandpaper. Auditory and tactile roughness have common features and evoke similar feelings for individuals. Moreover, smoother surfaces were perceived to be more pleasant while rougher surfaces were assessed as more unpleasant (Etzi et al., 2014), which is the same for sound preference. Inharmonic sounds are less preferred compared to harmonic sounds because people prefer consonance over dissonance (McDermott et al., 2010). Literature shows that abstract relationship between stimuli such as pleasantness or cognitive meaning may also help to establish cross-modal correspondence (Crisinel \& Spence, 2010). Furthermore, as Spence (2011) has suggested, some common - amodal - stimulus features between different sensory modalities (such as roughness in this study) may enhance the possibility of multisensory integration (Frings \& Spence, 2010; Radeau \& Bertelson, 1987; Thomas, 1941; Spence, 2007). Therefore, our findings also support that the abstract relationship between auditory and tactile stimuli in terms of roughness may also contribute to the audio-tactile interaction process for material perception.

In addition to abstract relationships, perceptual similarities during information processing may cause 'weak synesthesia,' which is also specified as 'cross-modal correspondence' (Spence \& Deroy, 2013). Common frequency channels between auditory and tactile sensory modalities in our brain (Yau et al., 2009) corroborate results that show similarities between auditory and tactile sensory processing. Further neuroimaging research is needed to understand how the brain processes frequency information obtained from harmonicity and surfaces. 
Our study and others studies (Jousmaki \& Hari, 1998; Guest et al., 2002, Yau et al. 2009) showed that there are crucial similarities between auditory and tactile sensory modalities in how their frequencies are processed by the brain. This brings up a new question: at which stage of information processing do these modalities interact and their featural information such as frequency is processed by the brain? (Butler et al., 2012). There are electrophysiological, neuroimaging, and anatomic tracing studies in humans and monkeys which demonstrate that auditory and somatosensory inputs interact in early sensory processing. It is also important to point out that even if higher-order multisensory integrative areas are damaged, the ability of multisensory integration can be still preserved (Foxe \& Schroeder, 2005). Although these studies draw attention to feedforward processing and early sensory interactions, other studies showed that sensory inputs may be processed independently by unimodal sensory systems. More specifically, feature-level information is independently extracted by these systems and later on combined in higher-order multisensory areas (Calvert et al., 1998; Driver and Noesselt, 2008). The electrophysiological study of Butler et al. (2012) shows that multisensory integration of auditory and tactile frequency information occurs in early sensory processing mechanisms preattentively to create frequency representation together. To investigate the same question with respect to audio-tactile roughness perception, our participants were presented with sequential and simultaneous harmonic and inharmonic sounds. According to our results, the interaction between auditory and tactile sensory inputs for roughness perception takes place in early processing due to the requirement of higher-level processing to perceive sequential harmonic and inharmonic sounds. However, this view needs to be supported with electrophysiological studies specifically for roughness perception.

Only three different roughness levels were used in this study which makes it difficult to generalize our results to all roughness levels that we encounter in our daily life. The same question should be investigated by using fine surfaces as well. Besides this, our harmonic sounds include fundamental frequency and its harmonics. However, consonant and dissonant sounds may have similar effects as harmonic and inharmonic sounds on tactile roughness perception. Consonant sounds are periodic just like harmonic sounds while dissonant sounds are perceived to be rough just like inharmonic sounds (Bidelman \& Heinz, 2011). Western tonal music can be used in further studies to examine the effects of auditory roughness on tactile roughness perception since consonant and dissonant sounds in Western tonal music have similar effects as harmonic and inharmonic sounds.

No sound condition affected roughness perception of surface types differently. While smooth surfaces were perceived rougher when there was no sound, medium surfaces were perceived smoother. We suspect that roughness perception of the smooth surfaces were confounded by the fact that this condition was presented as the first stimuli in the experiment, without any training session. This may cause the rating of smooth sandpaper to be higher. Since the roughness rating of medium surfaces relies on sound types and is prone to be perceived rougher with inharmonic sounds, mean roughness rating of the no-sound condition is already expected to be lower.

To summarize, our study shows that frequency representations in both auditory and tactile modalities could be dependent on their early-level interactions for roughness perception. These results also shed light on architectural design practices, which have started considering the auditory and tactile modalities as well as the visual modality in recent years. (Spence, 2020). Moreover, these results can be used for multisensory product design because people may construct an idea about the tactile properties of a product without touching it, like from auditory information (Spence \& Zampini, 2006). 


\section{Acknowledgement}

The authors acknowledge Ali Uslu for his proofreading assistance.

\section{References}

Altinsoy, M. E. (2008). The effect of auditory cues on the audiotactile roughness perception: Modulation frequency and sound pressure level. International Workshop on Haptic and Audio Interaction Design, 120-129.

Bidelman, G. M., \& Heinz, M. G. (2011). Auditory-nerve responses predict pitch attributes related to musical consonance-dissonance for normal and impaired hearing. The Journal of the Acoustical Society of America, 130(3), 1488-1502. https://doi.org/10.1121/1.3605559

Bieler, M., Xu, X., Marquardt, A., \& Hanganu-Opatz, I. L. (2018). Multisensory integration in rodent tactile but not visual thalamus. Scientific Reports, 8(1). https://doi.org/10.1038/s41598-018-33815-y

Butler, J. S., Foxe, J. J., Fiebelkorn, I. C., Mercier, M. R., \& Molholm, S. (2012). Multisensory Representation of Frequency across Audition and Touch: High Density Electrical Mapping Reveals Early Sensory-Perceptual Coupling. Journal of Neuroscience, 32(44), 15338-15344.

https://doi.org/10.1523/jneurosci.1796-12.2012

Calvert, G. A., Brammer, M. J., \& Iversen, S. D. (1998). Crossmodal identification. Trends in Cognitive Sciences, 2(7), 247-253. https://doi.org/10.1016/s1364-6613(98)01189-9

Carroll, A. B. (1970). The effect of harmonic and inharmonic frequency components on the perception of loudness and annoyance in complex sounds (Thesis). https://doi.org/10.21220/s2-8v42-3h86

Chimata, G., \& Schwartz, C. (2018). Investigation of the Role of Diminishing Surface Area on Friction-Based Tactile Discrimination of Textures. Biotribology, 15, 1-8. https://doi.org/10.1016/j.biotri.2018.07.001

Crisinel, A. S., \& Spence, C. (2010). A Sweet Sound? Food Names Reveal Implicit Associations between Taste and Pitch. Perception, 39(3), 417-425. https://doi.org/10.1068/p6574

Deroy, O., Crisinel, A. S., \& Spence, C. (2013). Crossmodal correspondences between odors and contingent features: odors, musical notes, and geometrical shapes. Psychonomic Bulletin \& Review, 20(5), 878-896. https://doi.org/10.3758/s13423-013-0397-0

Deroy, O., \& Spence, C. (2013). Why we are not all synesthetes (not even weakly so). Psychonomic Bulletin \& Review, 20(4), 643-664. https://doi.org/10.3758/s13423-013-0387-2

Driver, J., \& Noesselt, T. (2008). Multisensory Interplay Reveals Crossmodal Influences on 'Sensory-Specific' Brain Regions, Neural Responses, and Judgments. Neuron, 57(1), 11-23. https://doi.org/10.1016/j.neuron.2007.12.013

Ernst, M. O., \& Bülthoff, H. H. (2004). Merging the senses into a robust percept. Trends in Cognitive Sciences, 8(4), 162-169. https://doi.org/10.1016/j.tics.2004.02.002

Ernst, M. O., \& di Luca, M. (2011). Multisensory Perception: From Integration to Remapping. In J. Trommershauser \& K. Körding (Eds.), Sensory cue integration (pp. 224-250). Oxford University Press. https://doi.org/10.1093/acprof:oso/9780195387247.003.0012

Etzi, R., Spence, C., \& Gallace, A. (2014). Textures that we like to touch: An experimental study of aesthetic preferences for tactile stimuli. Consciousness and Cognition, 29, 178-188.

https://doi.org/10.1016/j.concog.2014.08.011

Foxe, J. J., Wylie, G. R., Martinez, A., Schroeder, C. E., Javitt, D. C., Guilfoyle, D., Ritter, W., \& Murray, M. M. (2002). Auditory-Somatosensory Multisensory Processing in Auditory Association Cortex: An fMRI Study. Journal of Neurophysiology, 88(1), 540-543. https://doi.org/10.1152/jn.2002.88.1.540 
Ghazanfar, A. A. (2005). Multisensory Integration of Dynamic Faces and Voices in Rhesus Monkey Auditory Cortex. Journal of Neuroscience, 25(20), 5004-5012. https://doi.org/10.1523/jneurosci.079905.2005

Guest, S., Catmur, C., Lloyd, D., \& Spence, C. (2002). Audiotactile interactions in roughness perception. Experimental Brain Research, 146(2), 161-171. https://doi.org/10.1007/s00221-002-1164-z

Heller, M. A. (1982). Visual and tactual texture perception: Intersensory cooperation. Perception \& Psychophysics, 31(4), 339-344. https://doi.org/10.3758/bf03202657

Jousmäki, V., \& Hari, R. (1998). Parchment-skin illusion: sound-biased touch. Current Biology, 8(6), R190-R191. https://doi.org/10.1016/s0960-9822(98)70120-4

Klatzky, R. L., \& Lederman, S. J. (1999). Tactile roughness perception with a rigid link interposed between skin and surface. Perception \& Psychophysics, 61(4), 591-607. https://doi.org/10.3758/bf03205532

Klatzky, R. L., \& Lederman, S. J. (2010). Multisensory texture perception. Multisensory Object Perception in the Primate Brain, 211-230. https://doi.org/10.1007/978-1-4419-5615-6_12

Koehler, S. D., Pradhan, S., Manis, P. B., \& Shore, S. E. (2011). Somatosensory inputs modify auditory spike timing in dorsal cochlear nucleus principal cells. European Journal of Neuroscience, 33(3), 409-420. https://doi.org/10.1111/j.1460-9568.2010.07547.x

Komura, Y., Tamura, R., Uwano, T., Nishijo, H., \& Ono, T. (2005). Auditory thalamus integrates visual inputs into behavioral gains. Nature Neuroscience, 8(9), 1203-1209. https://doi.org/10.1038/nn1528

Lakatos, P., Chen, C. M., O’Connell, M. N., Mills, A., \& Schroeder, C. E. (2007). Neuronal Oscillations and Multisensory Interaction in Primary Auditory Cortex. Neuron, 53(2), 279-292.

https://doi.org/10.1016/j.neuron.2006.12.011

Lederman, S. J. (1979). Auditory Texture Perception. Perception, 8(1), 93-103. https://doi.org/10.1068/p080093

Liew, K., \& Lindborg, P. (2017). Dissonance and roughness in cross-modal perception. Proceedings of the 6th Conference of the Asia Pacific Society for the Cognitive Sciences of Music.

https://www.researchgate.net/profile/KongmengLiew/publication/320620074_Dissonance_and_Roughness_in_Cross-

Modal_Perception/links/59f168ce0f7e9beabfca500b/Dissonance-and-Roughness-in-Cross-ModalPerception.pdf

Lindborg, P., \& Friberg, A. K. (2015). Colour Association with Music Is Mediated by Emotion: Evidence from an Experiment Using a CIE Lab Interface and Interviews. PLOS ONE, 10(12), e0144013. https://doi.org/10.1371/journal.pone.0144013

Love, J., Selker, R., Marsman, M., Jamil, T., Dropmann, D., Verhagen, J., Ly, A., Gronau, Q. F., Smíra, M., Epskamp, S., Matzke, D., Wild, A., Knight, P., Rouder, J. N., Morey, R. D., \& Wagenmakers, E. J. (2019). JASP: Graphical Statistical Software for Common Statistical Designs. Journal of Statistical Software, 88(2). https://doi.org/10.18637/jss.v088.i02

McDermott, J. H., Lehr, A. J., \& Oxenham, A. J. (2010). Individual Differences Reveal the Basis of Consonance. Current Biology, 20(11), 1035-1041. https://doi.org/10.1016/j.cub.2010.04.019

Murai, Y., \& Yotsumoto, Y. (2018). Optimal multisensory integration leads to optimal time estimation. Scientific Reports, 8(1). https://doi.org/10.1038/s41598-018-31468-5

Palmer, S. E., Schloss, K. B., Xu, Z., \& Prado-Leon, L. R. (2013). Music-color associations are mediated by emotion. Proceedings of the National Academy of Sciences, 110(22), 8836-8841. https://doi.org/10.1073/pnas.1212562110 
Parncutt, R., \& Hair, G. (2011). Consonance and dissonance in music theory and psychology: Disentangling dissonant dichotomies. Journal of Interdisciplinary Music Studies, 5(2). https://doi.org/10.4407/jims.2011.11.002

Pérez-Bellido, A., Anne Barnes, K., Crommett, L. E., \& Yau, J. M. (2018). Auditory Frequency Representations in Human Somatosensory Cortex. Cerebral Cortex, 28(11), 3908-3921. https://doi.org/10.1093/cercor/bhx255

Plack, C. J. (2010). Musical Consonance: The Importance of Harmonicity. Current Biology, 20(11), R476-R478. https://doi.org/10.1016/j.cub.2010.03.044

Ramachandran, V. S., \& Hubbard, E. M. (2001). Synaesthesia--a window into perception, thought and language. Journal of Consciousness Studies, 8(12), 3-34. https://doi.org/10.1111/1468-0068.00363

Ro, T., Ellmore, T., \& Beauchamp, M. S. (2013). A neural link between feeling and hearing. Cerebral Cortex, 23(7), 1724-1730. https://doi.org/10.1093/cercor/bhs166. $133-161$.

Schifferstein, H. N., \& Spence, C. (2008). Multisensory product experience. Product Experience,

Schneider, A., \& Frieler, K. (2008). Perception of harmonic and inharmonic sounds: Results from ear models. International Symposium on Computer Music Modeling and Retrieval, 18-44. https://doi.org/10.1007/978-3-642-02518-1_2

Schroeder, C. E., \& Foxe, J. (2005). Multisensory contributions to low-level, 'unisensory' processing. Current Opinion in Neurobiology, 15(4), 454-458. https://doi.org/10.1016/j.conb.2005.06.008

Schroeder, C. E., Lindsley, R. W., Specht, C., Marcovici, A., Smiley, J. F., \& Javitt, D. C. (2001). Somatosensory Input to Auditory Association Cortex in the Macaque Monkey. Journal of Neurophysiology, 85(3), 1322-1327. https://doi.org/10.1152/jn.2001.85.3.1322

Spence, C. (2011). Crossmodal correspondences: A tutorial review. Attention, Perception, \& Psychophysics, 73(4), 971-995. https://doi.org/10.3758/s13414-010-0073-7

Spence, C. (2020). Senses of place: architectural design for the multisensory mind. Cognitive Research: Principles and Implications, 5(1). https://doi.org/10.1186/s41235-020-00243-4

Spence, C., \& M, Z. (2006). Auditory contributions to multisensory product perception. Acta Acust. United Acust., 1009-1025.

Stein, B. E., \& Stanford, T. R. (2008). Multisensory integration: current issues from the perspective of the single neuron. Nature Reviews Neuroscience, 9(4), 255-266. https://doi.org/10.1038/nrn2331

Stevenson, R. A., Ghose, D., Fister, J. K., Sarko, D. K., Altieri, N. A., Nidiffer, A. R., Kurela, L. R., Siemann, J. K., James, T. W., \& Wallace, M. T. (2014). Identifying and Quantifying Multisensory Integration: A Tutorial Review. Brain Topography, 27(6), 707-730. https://doi.org/10.1007/s10548-014$0365-7$

Suzuishi, Y., Hidaka, S., \& Kuroki, S. (2020). Visual motion information modulates tactile roughness perception. Scientific Reports, 10(1). https://doi.org/10.1038/s41598-020-70831-3

Suzuki, Y., Gyoba, J., \& Sakamoto, S. (2008). Selective effects of auditory stimuli on tactile roughness perception. Brain Research, 1242, 87-94. https://doi.org/10.1016/j.brainres.2008.06.104

Tezcan Semerci, F. (2020). Recognition of sequential harmonic and inharmonic sound sequences investigated by behavioral and electrophysiological studies (Thesis).

https://acikbilim.yok.gov.tr/handle/20.500.12812/340584

Tramo, M. J., Cariani, P. A., Delgutte, B., \& Braida, L. D. (2001). Neurobiological foundations for the theory of harmony in western tonal music. Annals of the New York Academy of Sciences, 930(1), 92-116. https://doi.org/10.1111/j.1749-6632.2001.tb05727.x 
Xu, X., Hanganu-Opatz, I. L., \& Bieler, M. (2020). Cross-Talk of Low-Level Sensory and HighLevel Cognitive Processing: Development, Mechanisms, and Relevance for Cross-Modal Abilities of the Brain. Frontiers in Neurorobotics, 14. https://doi.org/10.3389/fnbot.2020.00007

Yau, J. M., DeAngelis, G. C., \& Angelaki, D. E. (2015). Dissecting neural circuits for multisensory integration and crossmodal processing. Philosophical Transactions of the Royal Society B: Biological Sciences, 370(1677). https://doi.org/10.1098/rstb.2014.0203

Yau, J. M., Hollins, M., \& Bensmaia, S. J. (2009). Textural timbre: The perception of surface microtexture depends in part on multimodal spectral cues. Communicative \& Integrative Biology, 2(4), 344 346. 